\title{
Analyses of the intermediate energy levels in ZnTe:O alloys
}

\author{
C. Tablero, a) A. Martí, and A. Luque \\ Instituto de Energía Solar, Universidad Politécnica de Madrid, Ciudad Universitaria s/n, \\ 28040 Madrid, Spain
}

\begin{abstract}
The isoelectronic doping of ZnTe with oxygen leads to deep levels on which carriers recombine radiatively via intermediate band states. The electronic density and the impurity-host character of these deep levels are analyzed using first principles, for the wurtzite and zinc-blende structures, different oxygen concentration, and different exchange-correlation approach.
\end{abstract}

The electronic properties of semiconductors can be modified through the substitution of a host atom with an isoelectronic impurity. The isoelectronic doping of II-VI compounds with oxygen has shown that oxygen gives rise to deep traps on which carriers recombine radiatively. ${ }^{1}$ As well as, some alloys, such as $\mathrm{ZnSe:O}$, have a reduced pressure coefficient. $^{2}$ It is explained in the framework of a band anticrossing between the states of the bottom of the ZnSe conduction band (CB) and a level or an intermediate band (IB) located $0.2 \mathrm{eV}$ above the bottom of the CB.

$O$ can be regarded as a shallow acceptor in II-VI semiconductors, but in $\mathrm{ZnTe}$ it was found to give rise to a deep level. Therefore, the ZnTe:O optoelectronic properties allow to expect a large optical gain in the intermediate emission band. The emission band lies about 0.4-0.6 eV (Refs. 3 and 4) below the CB. Additionally, two-photon operation at room temperature has been demonstrated with two light sources of different wavelength. ${ }^{3}$

Because of the technological importance of the ZnTe:O alloys as absorbent materials of solar radiation in solar cells, it is of much interest to explore the electronic properties, in particular, characterize the IB into the host band gap. In this letter we present some of the results obtained using first principles, in particular the analyses of the single-particle levels. These calculations are characterized by the following: (i) the local spin density approximation (LDA) and generalized gradient approximation (GGA) are used and compared, (ii) the two possible structures, the wurtzite (Ws) and zinc-blende (ZBs) are analyzed, (iii) cells from 32 to 256 atoms are employed. The number of atoms in the cell $(n)$ are related to the oxygen concentration as $\sim 3.5 \times 10^{22} / n \mathrm{~cm}^{-3}$ and $\sim 3.6$ $\times 10^{22} / n \mathrm{~cm}^{-3}$ for the $\mathrm{ZBs}$ and $\mathrm{Ws}$, respectively. Therefore, the simulations correspond to oxygen concentrations of $\sim 10^{20}-10^{21} \mathrm{~cm}^{-3}$.

In order to obtain the electronic properties, the standard Kohn-Sham (Ref. 5) equations are solved self-consistently ${ }^{6}$ using a confined pseudoatomic orbitals ${ }^{7}$ basis set. The LDA has been used with the Perdew-Zunger parametrization to the Ceperley-Alder numerical data, and in the form of Perdew, Burke, and Ernzerhof ${ }^{9}$ for GGA. The standard Troullier-Martins ${ }^{10}$ pseudopotential is adopted and expressed in the Kleinman-Bylander ${ }^{11}$ factorization.

The energy-band diagram for the $\mathrm{ZnTe}: \mathrm{O}$ and the $\mathrm{ZnTe}$ ZBs (with 64 atom-cell) around the $\Gamma$ point is shown in

\footnotetext{
${ }^{\text {a) }}$ Electronic mail: ctablero@etsit.upm.es.
}

Fig. 1, using GGA. By comparing the $\mathrm{ZnTe}: \mathrm{O}$ and the $\mathrm{ZnTe}$ band-structures, the substitution of the oxygen by Te drive to (i) a more complicated valence band (VB) edge structure, and (ii) a IB in the gap. The Fermi energy (not shown in the figure) is between the VB and the IB indicating that the IB has an acceptor character. As well as, in Fig. 2 is shown the band structures for the $\mathrm{ZnTe:O}$ around the $\Gamma$ point with LDA and GGA, for the ZBs (with 216 atom-cell) and the Ws (with a 108 and with a 256 atom-cell). The single-particle gap with ZBs-LDA $(\sim 2.2 \mathrm{eV})$ and with Ws-LDA $(\sim 2.0 \mathrm{eV})$ is lower that the ZBs-GGA $(\sim 2.51 \mathrm{eV})$ and the Ws-LDA $(\sim 2.4 \mathrm{eV})$ gaps. The experimental gap of the ZBs is $2.4 \mathrm{eV}$. Therefore, the results compare well with the experimental. From these figures, we see that (i) the GGA-gap is larger than the LDA-gap; (ii) the CB and the IB are wider for the LDA case; and (iii) an oxygen concentration increase pushes the IB deeper into the gap for the range of concentrations analyzed.

The contribution of the oxygen atom is mainly to the VB edge for all concentrations and structures analyzed, with both LDA and GGA. In fact, a population analyses using the Mulliken and Löwdin schemes leads to similar values for the atomic charges of the ZBs and Ws for different cell sizes, and with LDA and GGA. According to the Mulliken and Löwdin schemes, the oxygen increases +0.46 and +0.32 the number of electrons, respectively, whereas for $\mathrm{Te}$ atoms in $\mathrm{ZnTe}: \mathrm{O}$ the increment is +0.02 and +0.01 electrons, respectively. These differences are because the larger electronegativity of the oxygen atom with respect to Te atom. In order to analyze the charge redistribution when an oxygen atom is substituted by a Te atom, we obtain the radial charge $Q(r)$
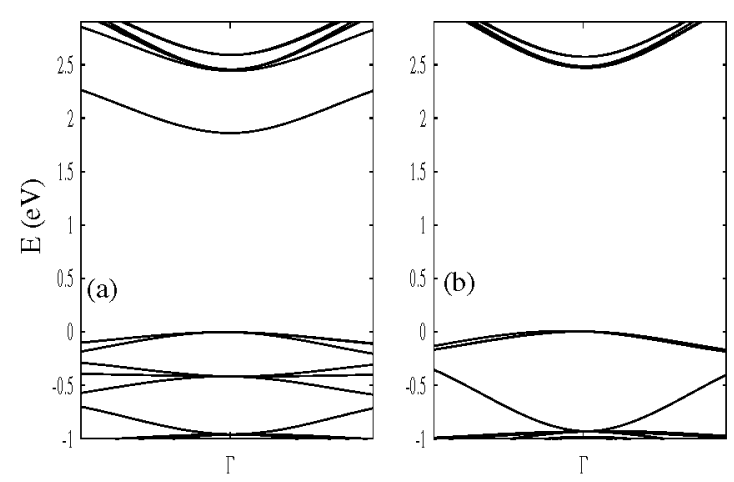

FIG. 1. (Color online) Energy-band diagram around the $\Gamma$ point in the $B Z$ with a 64-atom cell $\mathrm{ZBs}$ using GGA for (a) $\mathrm{ZnTe}: \mathrm{O}$ and (b) $\mathrm{ZnTe}$. 

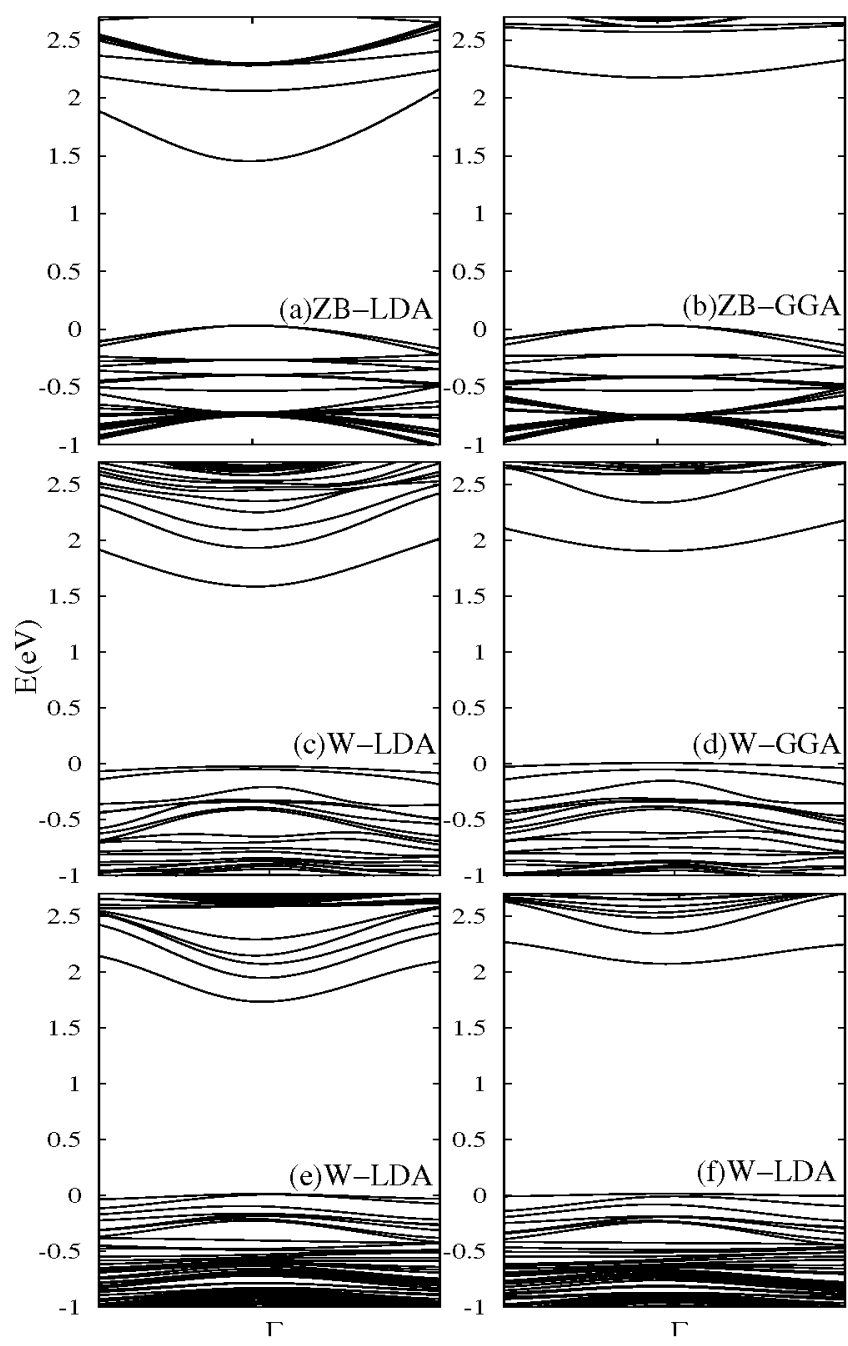

FIG. 2. (Color online) Energy-band diagram around the $\Gamma$ point in the $\mathrm{BZ}$ for ZnTe:O. (a) ZBs-LDA with a 216-atom cell; (b) ZBs-GGA with a 216atom cell; (c) Ws-LDA with a 108-atom cell; (d) Ws-GGA with a 108-atom cell; (e) Ws-LDA with a 256-atom cell; and (f) Ws-GGA with a 256-atom cell.

$=\int_{\Omega} \rho(\mathbf{r}) d \mathbf{r}$, where $\Omega_{r}$ is a sphere of radius $r$ centered on the oxygen atom and $\rho$ is the charge density. In Fig. 3 the difference between the radial charge of the $\mathrm{ZnTe}: \mathrm{O}$ and that of the host semiconductor is shown. At around $0.9 \AA$ from oxygen atom there is an appreciable charge accumulation with respect to the host semiconductor. For $2.62 \AA$ (vertical line in

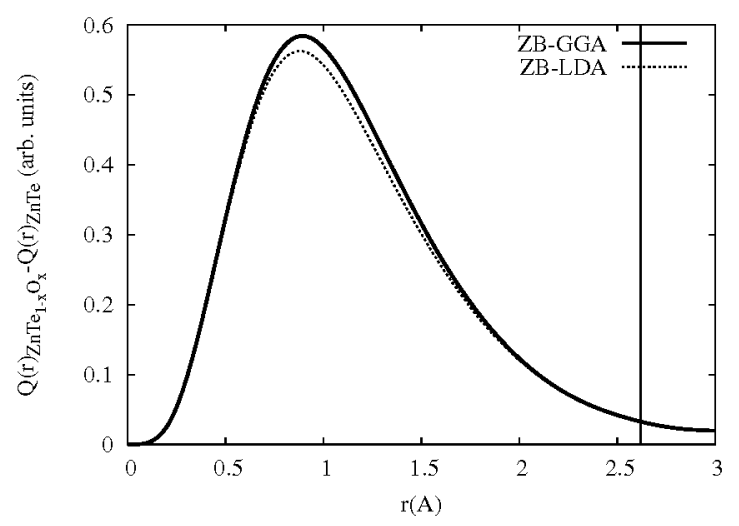

FIG. 3. (Color online) Difference between the radial charge $Q(r)$ of the $\mathrm{ZnTe}_{1-x} \mathrm{O}_{x}$ alloy and the host $\mathrm{ZnTe}$ semiconductor with $x=0.03$.
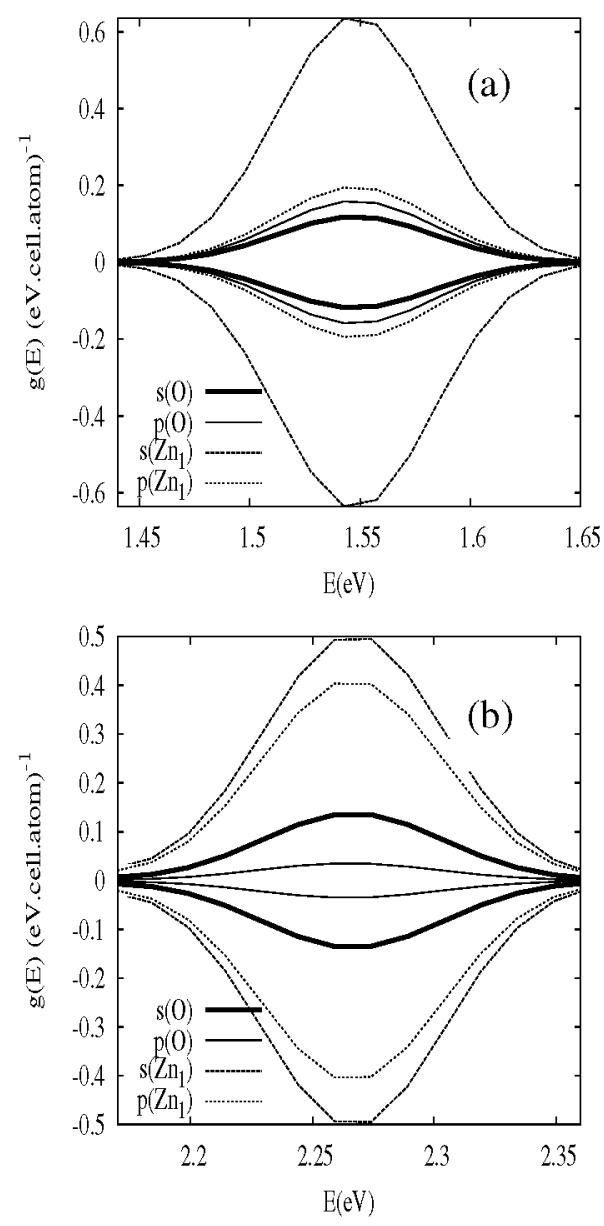

FIG. 4. (Color online) Projected DOS on the $s$ and $p$ orbitals of the oxygen and $\mathrm{Zn}_{1}$ atoms for (a) LDA-ZBs and (b) GGA-ZBs.

Fig. 3) there is a shell of four $\mathrm{Zn}$ atoms. For this distance the charge difference between the $\mathrm{ZnTe}: \mathrm{O}$ and $\mathrm{ZnTe}$ radial charge is small. From the figure, the difference between LDA and GGA is also small.

For the ZnTe host semiconductor, the Te site is tetrahedrally surrounded by four equivalent $\mathrm{Zn}$ atoms. However, when a Te atom is substituted by $\mathrm{O}$, the $\mathrm{Zn}$ atoms can be broken down into two following groups: those directly bonded to the oxygen atoms $\left(\mathbf{Z n}_{1}\right)$ and those which are not $\left(\mathrm{Zn}_{2}\right)$. The oxygen atoms will interact mainly with the $\mathrm{Zn}_{1}$ atoms. Therefore, the IB is created for the combination of the oxygen and the $\mathrm{Zn}_{1}$ orbitals. In order to identify the orbital composition of this band, the orbital projected density of states (DOS) of the oxygen and $\mathrm{Zn}_{1}$ atoms is represented in Fig. 4 for LDA-ZBs and GGA-ZBs. From the figure, it can also be seen that the oxygen and $\mathrm{Zn}_{1}$ orbitals that contribute most to the IB are the $s p$ orbitals. The $d$-O and $d-\mathrm{Zn}_{1}$ orbitals are not shown in the figure because its contribution is almost null. When GGA is used, the contribution of the $s$ - and $p-\mathrm{Zn}_{1}$ states is similar. However, the contribution of the $s-\mathrm{Zn}_{1}$ states is larger than the $p-\mathrm{Zn}_{1}$ states when LDA is used. Nevertheless, the difference between LDA and GGA is small. The linewidths of DOS from Fig. 4, and the bandwidths from the Figs. 1 and 2, indicate a sub gap structure due to the oxygen doping between $1.4-2.3 \mathrm{eV}$ depending of the oxygen concentration, structure, and exchange-correlation approach. This compares well with photoluminescence spectrums, ${ }^{3,4}$ 
where a strong subgap response related to oxygen doping in the range $1.6-2.0 \mathrm{eV}$ is observed.

The deep levels are considered as a negative effect because they act as Shockley, Read, and Hall recombination centers $^{12}$ via a lattice relaxation multiphonon emission mechanism. ${ }^{13}$ But it has also been shown that this negative effect can be avoided for charge redistribution between all the impurities ${ }^{14}$ and charge redistribution with the host semiconductor. ${ }^{15}$ Additionally, the solar cell devices with an IB have a theoretical limiting efficiency larger than conventional single-gap solar cells. ${ }^{16}$ However, for the $\mathrm{ZnTe} \mathrm{O}$, time-resolved photoluminescence measurements indicate a fast decay process from the $\mathrm{CB}$, and a slow radiative decay from the IB states. Measurements consisting of two-photon excitation further confirm transitions via IB band states. ${ }^{3}$ From the DOS analyses, the IB is made up of oxygen and $\mathrm{Zn}$ orbitals. Therefore, the low nonradiative recombination experimentally observed could be caused for the charge redistribution with the host semiconductor ${ }^{15}$ and between all the impurities. $^{14}$

Summarizing, the substitution of oxygen by Te in ZnTe leads to the formation of an IB located $0.4-0.6 \mathrm{eV}$ below the $\mathrm{CB}$ according to experimental results. This $\mathrm{IB}$ is mainly made up of the oxygen and for the $\mathrm{Zn}$ nearest neighbors. These results happen with the different oxygen concentrations analyzed, different structures (Ws and ZBs), and different calculation scheme (LDA and GGA). The analyses of the composition of the IB could justify the low nonradiative recombination observed experimentally.

This work has been supported by the GENESIS FV project of the National Spanish program CONSOLIDER (Grant No. CSD2006-0004), by the European Commission through the funding of the project IBPOWER (Ref. No. Grant Agreement 211640), and by La Comunidad de Madrid through the funding of the project NUMANCIA-2 (Ref. No. S-2009/ENE-1477).

${ }^{1}$ J. L. Merz, Phys. Rev. 176, 961 (1968); W. K. Ge, S. B. Lam, I. K. Sou, J. Wang, Y. Wang, G. H. Li, H. X. Han, and Z. P. Wang, Phys. Rev. B 55, 10035 (1997); M. J. Seong, H. Alawadhi, I. Miotkowski, A. K. Ramdas, and S. Miotkowska, ibid. 60, R16275 (1999); Y.-M. Yu, S. Nam, K.-S. Lee, Y. Dae Choi, and O. Byungsung, J. Appl. Phys. 90, 807 (2001).

${ }^{2}$ W. Shan, W. Walukiewicz, J. W. Ager III, K. M. Yu, J. Wu, E. E. Haller, Y. Nabetani, T. Mukawa, Y. Ito, and T. Matsumoto, Appl. Phys. Lett. 83, 299 (2003).

${ }^{3}$ W. Wang, A. S. Lin, J. D. Phillips, and W. K. Metzger, Appl. Phys. Lett. 95, 261107 (2009).

${ }^{4}$ Y. Burki, P. Sshwendimann, W. Czaja, and H. Berger, J. Phys.: Condens. Matter 5, 9235 (1993); Europhys. Lett. 13, 555 (1990).

${ }^{5}$ W. Kohn and L. J. Sham, Phys. Rev. 140, A1133 (1965).

${ }^{6}$ J. M. Soler, E. Artacho, J. D. Gale, A. García, J. Junquera, P. Ordejon, and D. Sánchez-Portal, J. Phys.: Condens. Matter 14, 2745 (2002), and references therein.

${ }^{7}$ O. F. Sankey and D. J. Niklewski, Phys. Rev. B 40, 3979 (1989).

${ }^{8}$ J. P. Perdew and A. Zunger, Phys. Rev. B 23, 5048 (1981); D. M. Ceperley and B. J. Alder, Phys. Rev. Lett. 45, 566 (1980).

${ }^{9}$ J. P. Perdew, K. Burke, and M. Ernzerhof, Phys. Rev. Lett. 77, 3865 (1996); 78, 1396 (1997).

${ }^{10}$ N. Troullier and J. L. Martins, Phys. Rev. B 43, 1993 (1991).

${ }^{11}$ L. Kleinman and D. M. Bylander, Phys. Rev. Lett. 48, 1425 (1982); D. M. Bylander and L. Kleinman, Phys. Rev. B 41, 907 (1990).

${ }^{12}$ W. Shockley and W. T. Read, Phys. Rev. 87, 835 (1952); R. N. Hall, ibid. 87, 387 (1952).

${ }^{13}$ D. V. Lang and C. H. Henry, Phys. Rev. Lett. 35, 1525 (1975); K. V. Boer, Survey of Semiconductor Physics (Wiley, New York, 2002).

${ }^{14}$ A. Luque, A. Martí, E. Antolín, and C. Tablero, Physica B 382, 320 (2006).

${ }^{15}$ C. Tablero, Physica B 404, 4023 (2009).

${ }^{16}$ A. Luque and A. Martí, Phys. Rev. Lett. 78, 5014 (1997). 